\title{
Delineation of retroperitoneal metastatic lymph nodes in ovarian cancer with near-infrared fluorescence imaging
}

\author{
TAO PU ${ }^{1 *}$, LIQIN XIONG $^{2 *}$, QIYU LIU ${ }^{1}$, MINXING ZHANG $^{1}$, QINGQING CAI $^{3}$,

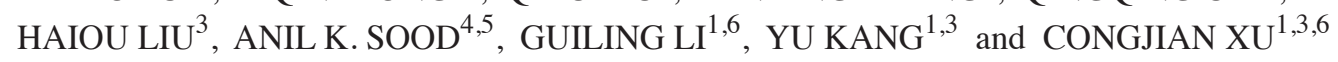 \\ ${ }^{1}$ Department of Obstetrics and Gynecology, Shanghai Medical School, Fudan University, Shanghai 200032; \\ ${ }^{2}$ School of Biomedical Engineering, Med-X Research Institute, Shanghai Jiao Tong University, Shanghai 200030; \\ ${ }^{3}$ Shanghai Key Laboratory of Female Reproductive Endocrine Related Diseases, Shanghai 200011, P.R. China; \\ Departments of ${ }^{4}$ Gynecologic Oncology and Reproductive Medicine and ${ }^{5}$ Cancer Biology, Center for RNAi \\ and Non-Coding RNA, The University of Texas MD Anderson Cancer Center, Houston, Texas 77030, USA; \\ ${ }^{6}$ Department of Integrated Traditional Chinese and Western Medicine, Obstetrics \\ and Gynecology Hospital, Fudan University, Shanghai 200011, P.R. China
}

Received October 3, 2015; Accepted March 14, 2017

DOI: $10.3892 / 01.2017 .6521$

\begin{abstract}
Lymph node metastasis occurs in early-stage and late-stage ovarian cancers. Systematic lymphadenectomy is frequently conducted in an attempt to prevent disease progression. However, this method is associated with multiple complications. Therefore, it is necessary to develop a less invasive and more sensitive method for detecting lymphatic metastasis in ovarian cancer. The aim of the present study was to develop an appropriate fluorescent label for the analysis of lymphatic metastasis in vivo. To this end, epithelial ovarian cancer cells with high potential for lymph node metastasis were labeled using mCherry fluorescence. The cells were then imaged in vitro to determine the expression of mCherry, and in a mouse xenograft model in vivo. The data demonstrated the successful identification of metastatic retroperitoneal lymph nodes by co-localization with lymph nodes labeled by near-infrared fluorescence nanoparticles in vivo. These data
\end{abstract}

Correspondence to: Dr Yu Kang or Professor Guiling Li, Department of Obstetrics and Gynecology, Shanghai Medical School, Fudan University, 138 Yixueyuan Road, Shanghai 200032, P.R. China

E-mail: yukang@fudan.edu.cn

E-mail: guilingli@fudan.edu.cn

*Contributed equally

Abbreviations: NIR, near-infrared; MEH-PPV, poly (2-methoxy -5-[2-ethylhexyloxy]-1, 4-phenylenevinylene); MW, molecular weight; THF, tetrahydrofluoride; UV, ultraviolet; H\&E, hematoxylin and eosin; IHC, immunohistochemistry; CK, cytokeratin; PET, positron emission tomography; HER-2, human epidermal growth factor receptor 2; FR, folate receptor; ICG, indocyanine green

Key words: ovarian cancer, near-infrared fluorescence imaging, metastatic cancer, nanoparticles, retroperitoneal lymph nodes provided important insights into the further development of methods for intra-operative identification of lymphatic metastasis and the mechanisms underlying lymphatic metastasis.

\section{Introduction}

Ovarian cancer is a clinically significant health problem globally. Estimates suggested that there would be $>20,000$ new cases of ovarian cancer and $\sim 15,000$ mortalities due to ovarian cancer in the United States in 2015 (1). Tumor cells spread by direct extension, transcoelomic dissemination, lymphatic involvement and, occasionally, via the hematogenous route (2-4). According to FIGO staging, cancers involving the retroperitoneal lymph nodes are classified as stage III cancers (5). However, systematic lymphadenectomy has demonstrated that lymph node metastasis also occurs during the early stages (stages I and II) in $14.2 \%$ of all patients (6). Systematic lymphadenectomy, which is predominantly performed in the para-aortic and pelvic regions $(6,7)$, is associated with multiple complications and is considered quite invasive $(8,9)$. The most frequent complications associated with retroperitoneal lymphadenectomy include postoperative mortality, vascular injury, lymphocyst formation, deep venous thrombosis and pulmonary embolism (10-12). Therefore, there is a need for the development of less invasive and more sensitive methods for the detection of lymphatic metastasis in ovarian cancer. Several models for ovarian cancer are commonly used, including xenografts, genetically engineered mouse models, laying hen models, ovarian cancer stem cells and three-dimensional culture models (13-15). Among these models, xenografts are the most common due to the high tumor rates, relatively low cost and short experimental period. Intraperitoneal, subcutaneous, and orthotopic implantation methods are conventional techniques for establishing ovarian tumors and have been adapted to mimic different clinical scenarios.

However, despite its importance for cancer diagnosis and prognosis, preclinical evaluation of lymph node metastasis is 
difficult in small animals (16). The aim of the present study was to develop an appropriate model for studying metastatic ovarian cancer localized in the retroperitoneal lymph nodes using near-infrared (NIR) fluorescence imaging in a xenograft mouse model. These data are expected to provide insights into the further applications of intra-operative identification of lymphatic metastasis and studies of the mechanisms of lymphatic metastasis.

\section{Materials and methods}

Cell lines and animals. SKOV-3 cells, established from a human ovarian adenocarcinoma, were obtained from the Shanghai Institute of Cell Biology of the Chinese Academy of Sciences (Shanghai, China). The SKOV-3-LN subline, which has the potential to induce higher rates of lymphatic metastasis, peritoneal dissemination and bloody ascites, was established previously in our laboratory (17). Cells were grown in McCoy's 5A medium (Hyclone; GE Healthcare Life Sciences, Logan, UT, USA) and were supplemented with $10 \%$ (v/v) fetal bovine serum (Thermo Fisher Scientific, Inc., Waltham, MA, USA), $100 \mathrm{U} / \mathrm{ml}$ penicillin, and $100 \mu \mathrm{g} / \mathrm{ml}$ streptomycin. Cells were maintained at $37^{\circ} \mathrm{C}$ in a humidified incubator containing $5 \%$ $\mathrm{CO}_{2}$. Cultures were passaged every 3 days.

Female BALB/c nude mice (7-8 weeks old) were purchased from the Animal Center of the Chinese Academy of Sciences (Shanghai, China) and maintained under specific pathogen-free conditions at the Department of Laboratory Animal Science, Fudan University (Shanghai, China). A total of 6 mice (weighing $20 \mathrm{~g}$ ) were used in the present study, they were raised in an atmosphere of $22 \pm 2^{\circ} \mathrm{C}$ with $65 \pm 5 \%$ humidity, with a $12 \mathrm{~h}$ light-dark cycle and ad libitum access to water and food. The present study was performed with the approval of the Animal Ethics Committee of the Obstetrics and Gynecology Hospital, Fudan University and in accordance with the Guide for the Ethical Treatment of Laboratory Animals from the Ministry of Science and Technology of the People's Republic of China (publication no. 2006-398).

Synthesis of NIR nanoparticles. Nanoparticles with a high lymph node labeling efficiency were synthesized as follows. The poly (phenylenevinylene) derivative poly [2-methoxy-5-(2-ethylhexyloxy)-1, 4-phenylenevinylene; MEH-PPV; molecular weight (MW): 150,000-250,000 Da] was purchased from J\&K, Inc. (Beijing, China). PS-PEG-COOH (MW: 21,700 Da for the PS moiety; 1,200 Da for PEG-COOH; polydispersity, 1.25) was purchased from Polymer Source, Inc. (Montreal, Canada). Silicon 2, 3-naphthalocyanine bis (trihexylsilyloxide) (NIR775) was purchased from Sigma Aldrich; Merck KGaA (Darmstadt, Germany). NIR775-doped NIR nanoparticles were prepared as previously described (18). In a typical procedure, a solution of tetrahydrofluoride (THF) containing $125 \mu \mathrm{g} / \mathrm{ml}$ MEH-PPV, $125 \mu \mathrm{g} / \mathrm{ml}$ PS-PEG-COOH, and $1.5 \mu \mathrm{g} / \mathrm{ml} \mathrm{NIR775}$ dye was prepared. An aliquot of the mixture $(2 \mathrm{ml})$ was then dispersed into $10 \mathrm{ml}$ water under vigorous sonication. Extra THF was evaporated at an elevated temperature (below $90^{\circ} \mathrm{C}$ ) under the protection of nitrogen. Sizes and morphologies of NIR nanoparticles were determined using a Tecnai G2 Spirit Bio-twin transmission electron microscope (TEM; Thermo Fisher Scientific, Inc.) at $120 \mathrm{kV}$. TEM samples were prepared by dripping the nanoparticle solution $(5 \mu \mathrm{l}, 500 \mu \mathrm{g} / \mathrm{ml})$ onto a carbon-supported copper grid and allowed to dry at room temperature prior to observation. The grid can then be directly observed using a TEM following evaporation of the water (18). The absorption spectra were recorded on a Shimadzu UV-2550 ultraviolet (UV)-Vis spectrometer (Shimazdu Corporation, Kyoto, Japan). Fluorescence emission spectra were collected with an Edinburgh LFS-920 spectrophotometer (Edinburgh Instruments, Ltd., Livingston, UK). Particles were concentrated (3 min/2,685.6 $\mathrm{x} g$ at room temperature) using Amicon Ultra $4 \mathrm{ml}$ centrifugal filters with a membrane nominal molecular weight limit of $50 \mathrm{kDa}$ (Merck KGaA) for in vivo injection and imaging.

Generation and analysis of fluorescently labeled epithelial ovarian cancer (EOC) cells using lentivirus. SKOV-3-LN cells were infected with lentivirus (LV-GIDL32812-RNAi (14974-1; Genechem Co., Ltd., Shanghai, China) containing an $11.4 \mathrm{~kb}$ vector for the mCherry sequence (GV298 Genechem Co., Ltd.) and a puromycin resistance gene grown in McCoy's 5A medium with extra $5 \mu \mathrm{g} / \mathrm{ml}$ polybrene (Genechem Co., Ltd.) added for lentivirus infection enhancement during infection for $16 \mathrm{~h}$. Infection continued by replacing the medium back to normal McCoy's 5A medium for another $72 \mathrm{~h}$. Clones infected with a blank control lentivirus was used for a negative control. Clones were selected in medium containing puromycin $(1 \mu \mathrm{g} / \mathrm{ml})$ for $48 \mathrm{~h}$. During infection, cells were maintained at $37^{\circ} \mathrm{C}$ in a humidified incubator containing $5 \% \mathrm{CO}_{2}$. Fluorescently-labeled EOC cells were named SKOV3-M and SKOV3-LN-M cells. Cultured SKOV3-LN and SKOV3-LN-M cells were trypsinized, washed in PBS, and observed by fluorescence microscope (Nikon ECLIPSE Ti; NIS-Elements software v. 4.0; Nikon Corporation, Tokyo, Kanto, Japan). Analysis for mCherry expression was performed by fluorescence-activated cell sorting (FACS) at a cell density of $10^{5}$ cells/ml using the FL3 channel on a FACS Caliber instrument (BD Biosciences, San Jose, CA, USA) and FCSExpress V3.1 software (De Novo Software, Glendale, CA, USA). Cells at different densities $\left(2 \times 10^{5}\right.$, $1 \times 10^{5}, 5 \times 10^{4}$ and $2.5 \times 10^{4}$ cells/well) were imaged in 96-well plates using an IVIS Spectrum Imaging System (PerkinElmer, Inc., Waltham, MA, USA).

Establishment of tumor xenografts and in vivo imaging. Cultured SKOV3-LN-M cells were trypsinized, washed in PBS and resuspended in Hanks' Balanced Salt Solution (Thermo Fisher Scientific, Inc.). Next, $1 \times 10^{6}$ cells in a $30 \mu \mathrm{l}$ volume were injected into the left ovary of mice. A total of four mice were injected and imaged. Mice were imaged using excitation/emission 587/610 nm filters for detection of the mCherry fluorescence signal and using excitation/emission 465/780 nm filters for detection of the nanoparticle fluorescence signal in situ using the IVIS Spectrum System (PerkinElmer, Inc.) 5 weeks following injection. A total of $\sim 50 \mu \mathrm{g}$ nanoparticles were delivered by tail veil injection $24 \mathrm{~h}$ prior to imaging, and the mice were fasted to achieve the maximum decrease in autofluorescence. The mice were then sacrificed via cervical dislocation, and images were analyzed using Living Imaging software v. 4.4 (PerkinElmer, Inc.).

Once the imaging was completed, retroperitoneal lymph nodes were harvested, preserved in $4 \%$ paraformaldehyde, 
A

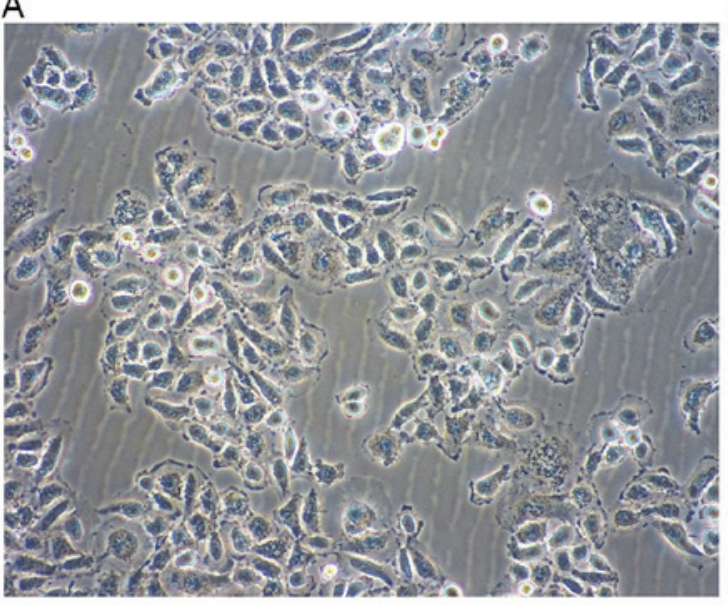

C

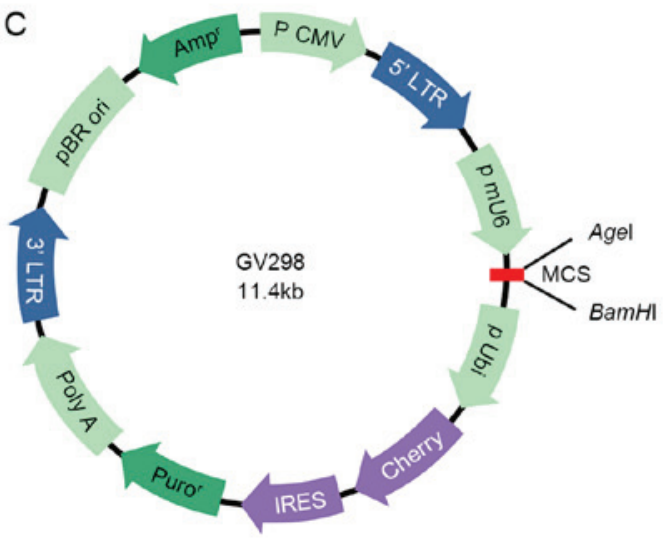

E

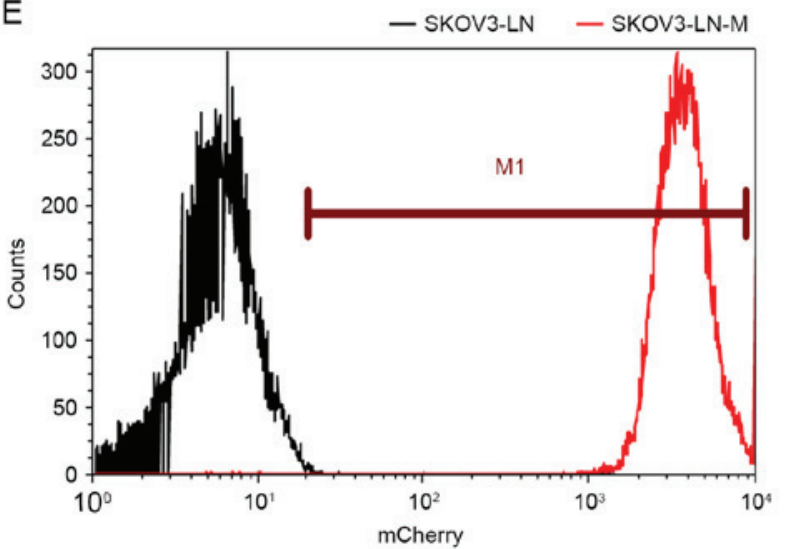

B

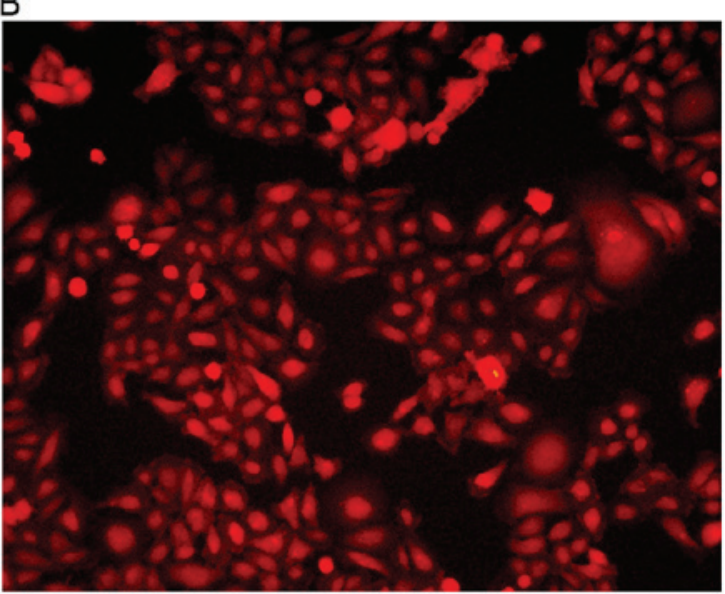

D

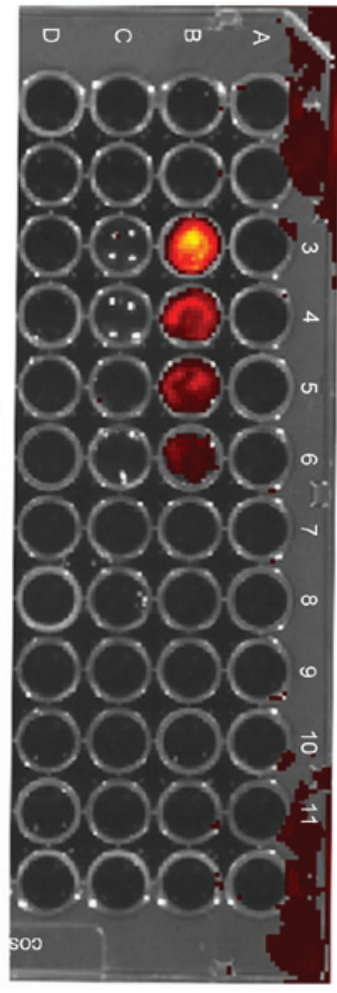

Epi-fluorescence

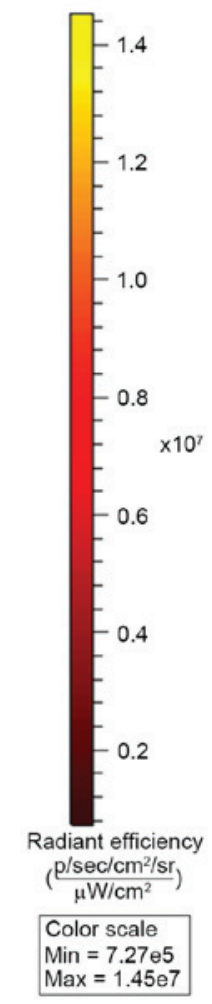

Figure 1. Morphology and signal gradient of fluorescently labeled epithelial ovarian cancer. Cells were visualized under a fluorescence microscope (A) without and (B) with a fluorescence filter (x100 magnification). (C) An $11.4 \mathrm{~kb}$ vector for lentivirus fluorescent labeling. (D) Fluorescence signal gradient detected using the IVIS Spectrum imaging system (column B), with negative control (column C). (E) Fluorescence-activated cell sorting analysis of mCherry fluorescence. LTR, long terminal repeat; Amp', ampicillin; p CMV, cytomegalovirus promotor; p mU6, mouse U6 promotor; $p$ Ubi, ubiquitin promotor; IRES, internal ribosome entry site; Puro', puromycin.

sectioned (4 $\mu \mathrm{m}$ thick) for subsequent hematoxylin and eosin (H\&E) staining and immunohistochemistry (IHC) for the detection of human cytokeratin (CK) 8 and 7. As the para-aortic lymph nodes are the most common metastatic nodes, imaging and pathological verification were limited to these lymph nodes. In IHC staining, sections were de-waxed and rehydrated after being heated at $60^{\circ} \mathrm{C}$ for $1 \mathrm{~h}$. Antigen retrieval was performed by incubation of the slides with EDTA (PH 9.0; Wuhan Boster Biological Technology Ltd., Wuhan, China) at $100^{\circ} \mathrm{C}$ for $30 \mathrm{~min}$. Following cooling to room temperature, endogenous peroxidase blocking was performed by incubation with $3 \%$ hydrogen peroxidase for $25 \mathrm{~min}$ in the dark at room temperature and $3 \%$ bovine serum albumin (Beijing Solarbio Science \& Technology, Co., Ltd., Beijing, China) was used for background blocking at room temperature for $30 \mathrm{~min}$. Incubation with primary antibody anti-human CK7 (1:100; clone OV-TL 12/30; Dako; Agilent Technologies, Inc., Santa Clara, CA, USA; cat. no. M7018) or anti-human CK8 (1:100; clone TS-1; Thermo Fisher Scientific, Inc.; cat. no. MA5-14428) was performed overnight at $4^{\circ} \mathrm{C}$. Subsequently, the slides were incubated with peroxidase-conjugated anti-mouse $\operatorname{IgG}$ (1:1; Dako; Agilent Technologies, Inc.; cat. no. K5007) for $50 \mathrm{~min}$ at room 
A

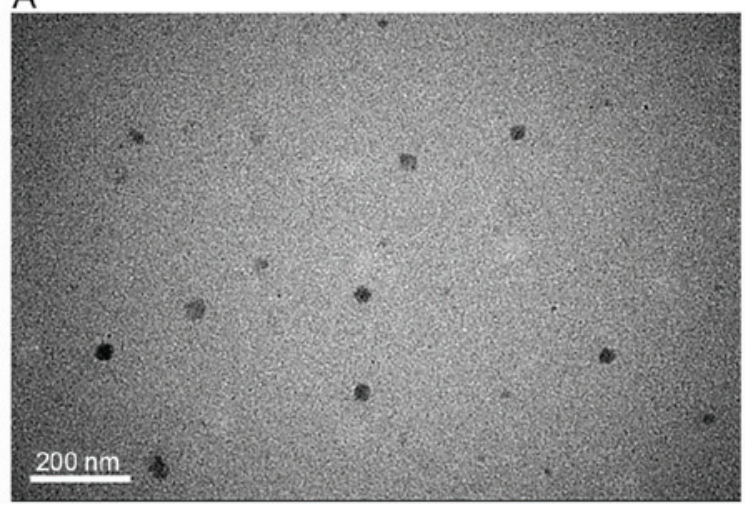

B

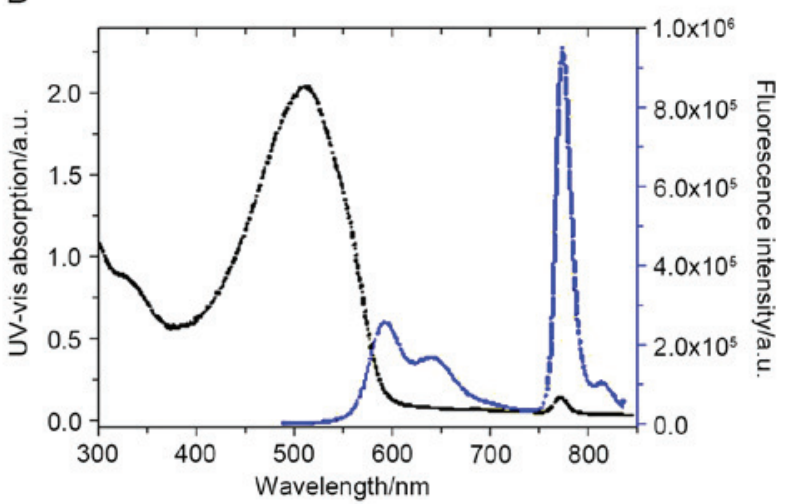

C

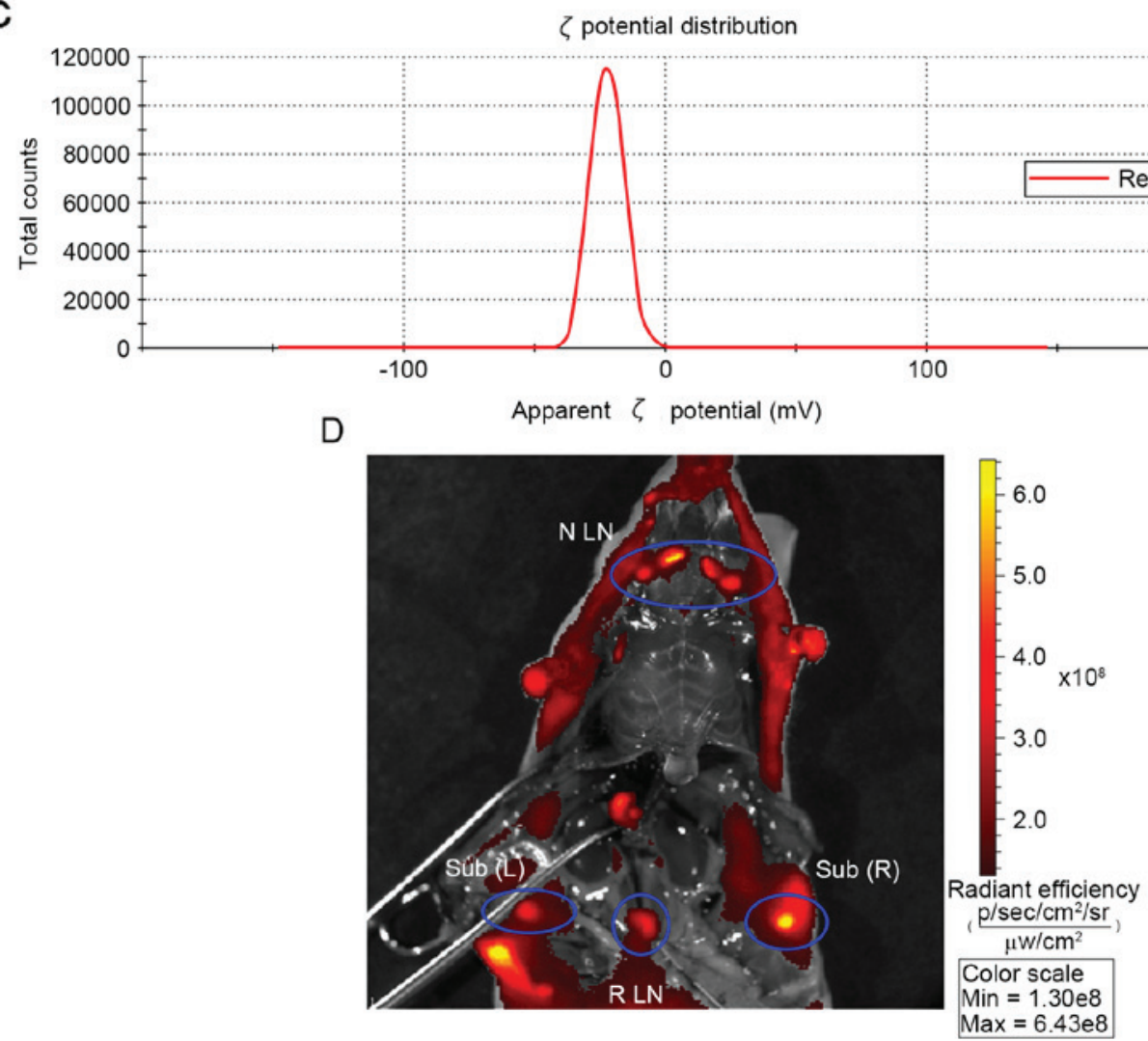

Figure 2. Morphology, parameters and localization of NIR nanoparticles. (A) Average diameter of NIR nanoparticles, determined using transmission electron microscopy. (B) Excitation and emission parameters of NIR nanoparticles. (C) $\zeta$ potential analysis of NIR nanoparticles. (D) Localization of the lymph nodes with nanoparticles (in blue circles). Field of view, 10x10 $\mathrm{cm}^{2}$. NIR, near-infrared; UV, ultraviolet; N LN, lymph nodes in the neck region; Sub (L), subiliac lymph nodes in the left side; Sub (R), subiliac lymph nodes in the right side; R LN, retroperitoneal lymph nodes.

temperature followed by staining with diaminobenzidine and nuclei counter-stain at room temperature for $10 \mathrm{sec}$ each. The results of $\mathrm{H} \& \mathrm{E}$ staining and $\mathrm{IHC}$ were observed and carefully checked under a Leica DM2500 Microscope by at least two pathologists independently, using Leica LAS v. 4.2 software (Leica Microsystems GmbH, Wetzlar, Hesse, Germany).

\section{Results}

In vitro fluorescence of cancer cells. Fluorescently-labeled EOC cells were imaged to observe mCherry fluorescence. SKOV3-LN cells grew in clusters, and SKOV3-LN-M cells exhibited strong fluorescence. FACS analysis revealed that $99.8 \%$ of SKOV3-LN cells were labeled with mCherry.
Furthermore, images of cells at different densities revealed that the emitted fluorescence signals varied according to the cell density in SKOV3-LN-M cells. At the least, $2.5 \times 10^{4}$ cells were detected in vivo. In contrast, no fluorescence signal was detected in SKOV3-LN cells (Fig. 1).

Characterization of NIR nanoparticles. TEM images revealed that the nanoparticles were dispersed with an average diameter of $\sim 23 \mathrm{~nm}$ (Fig. 2A). The NIR nanoparticles exhibited a broad UV-Vis band with a maximum at $510 \mathrm{~nm}$ (Fig. 2B). Under excitation at $480 \mathrm{~nm}$, these nanoparticles exhibited weak MEH-PPV emission at $595 \mathrm{~nm}$, but a strong NIR peak at $776 \mathrm{~nm}$. $\zeta$ potential analysis predicted that the NIR nanoparticles had a $\zeta$ potential $>-30 \mathrm{mV}$ (Fig. 2C). Identification of the 
A

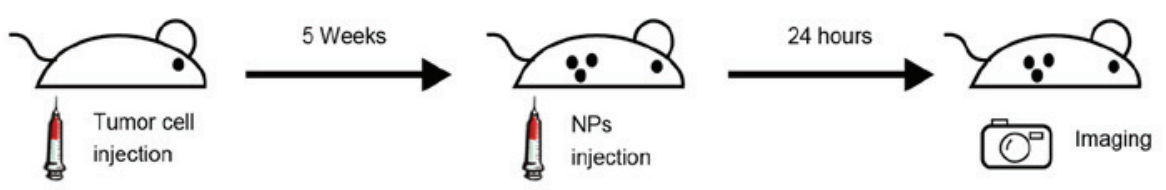

B

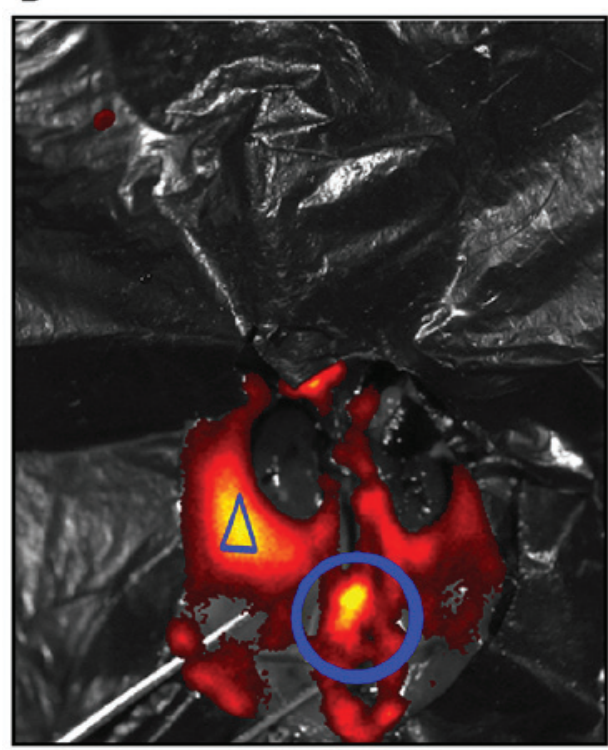

D

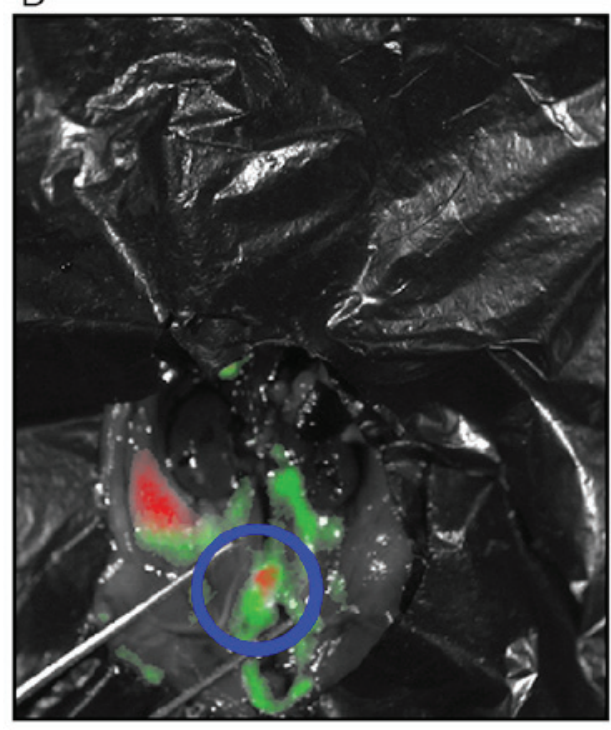

Epi-fluorescence

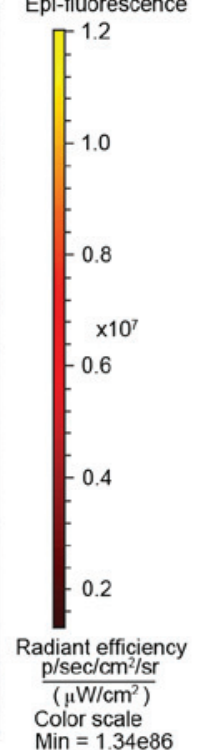

$\operatorname{Max}=1.20 \mathrm{e} 7$

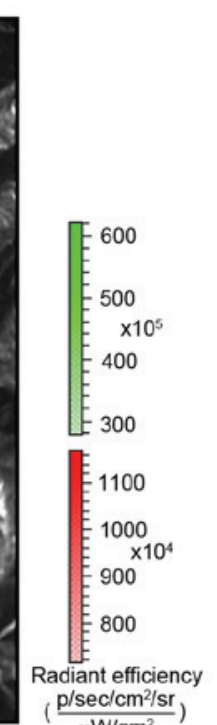

C

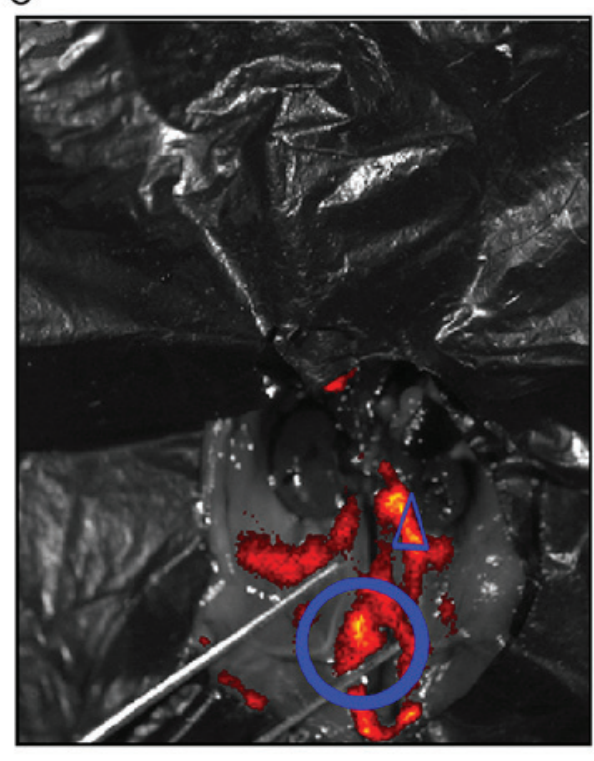

Epi-fluorescence

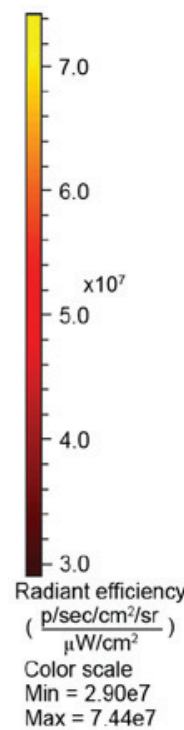

E

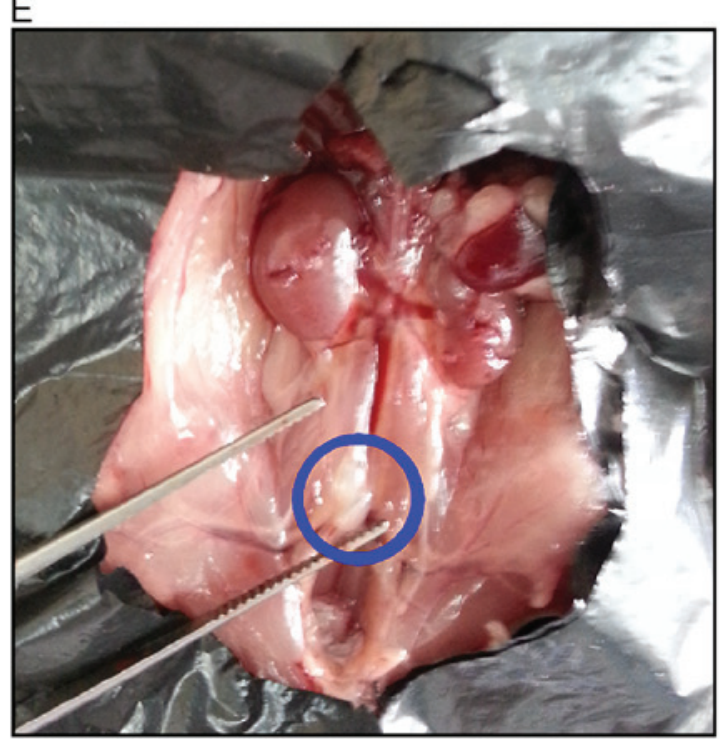

Figure 3. MCherry and nanoparticle fluorescent signals in the mouse model. (A) Flowchart of metastatic retroperitoneal lymph node imaging in the xenograft mouse model. (B) Representative image of the fluorescence signal of mCherry for tumor cells (blue circle and blue triangle). (C) Representative image of the fluorescence signal of nanoparticles (blue circle) for lymph nodes and potential lymphatic vessels (blue triangle). (D) Spatial overlapping signals for the localization of metastatic lymph nodes (red, mCherry signals; green, nanoparticle signals; indicated by the blue circle) using light spectrum unmixing software. (E) Corresponding gross anatomy of the retroperitoneal lymph nodes. Field of view, $10 \times 10 \mathrm{~cm}^{2}$.

lymph nodes was evaluated using an in vivo imaging system. Major lymph nodes, including lymph nodes in the neck region, subiliac lymph nodes and the retroperitoneal lymph nodes, were visualized (Fig. 2D).

In vivo imaging of tumor xenografts. Tumor xenografts were established and imaged as illustrated in the flowchart in Fig. 3A and described in the materials and methods section. Tumor cells exhibited a strong and well-defined fluorescent signal for mCherry (Fig. 3B). Notably, the fluorescence signal of the nanoparticles was observed in the lymph nodes and adjacent potential lymphatic vessels (Fig. 3C). Spectral unmixing analysis demonstrated that the mCherry and nanoparticle signals overlapped, suggesting that metastatic lymph nodes were detected (Fig. 3D). The corresponding gross anatomy of the retroperitoneal lymph nodes is depicted in Fig. 3E.

H\&E staining was then performed on the lymph nodes identified as potentially metastatic. However, the imaging-positive lymph nodes tested negative following histopathological examination (Fig. 4A and B). Therefore, IHC was performed 


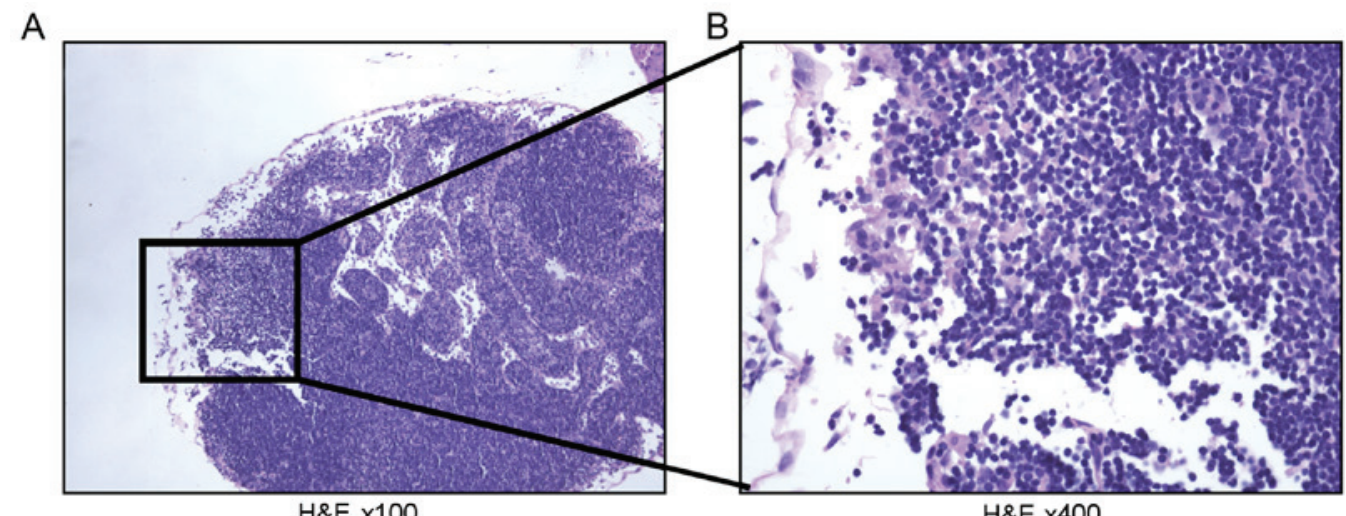

C

$\mathrm{D}$

$\mathrm{H} \& \mathrm{E} \times 400$

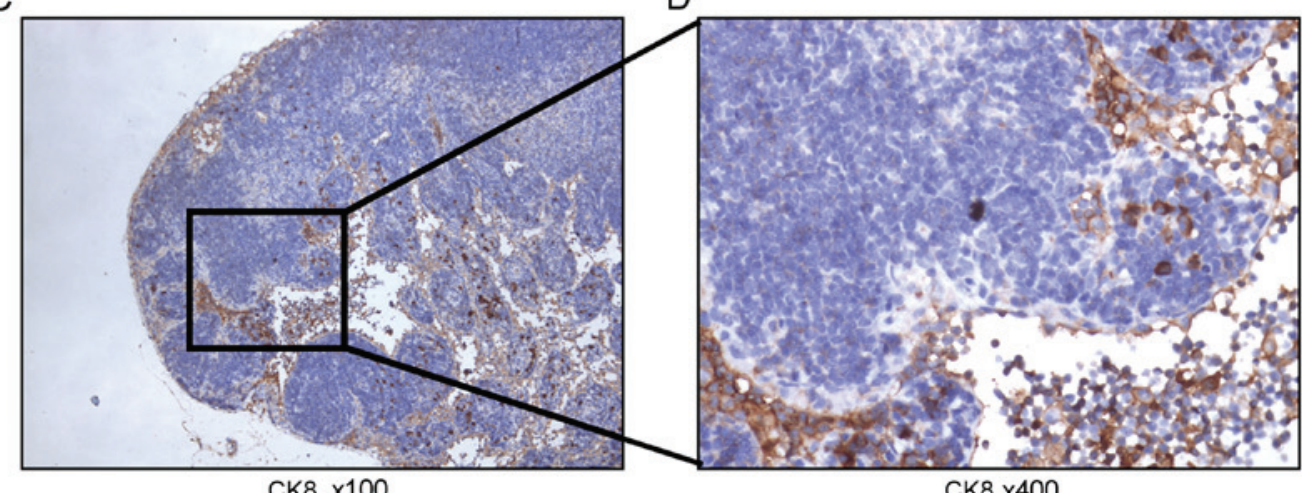

E

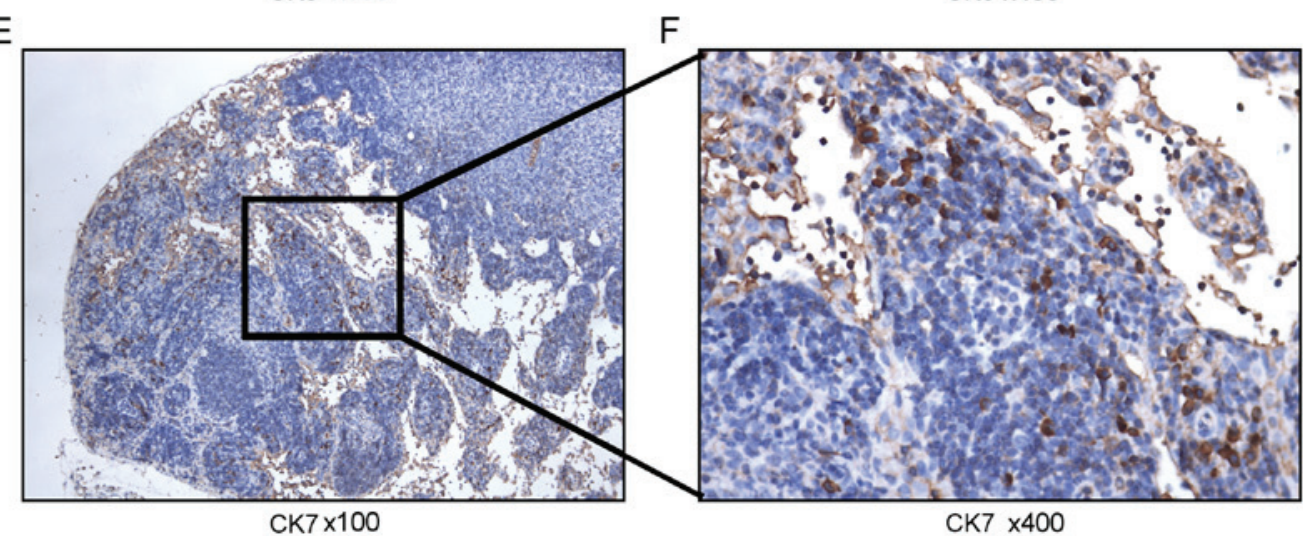

Figure 4. Staining of imaging-positive lymph nodes. H\&E staining of imaging-positive lymph nodes at (A) x100 and (B) x400 magnification. IHC analysis of human CK8 in imaging-positive lymph nodes at (C) x100 and (D) x400 magnification. IHC analysis of human CK7 in imaging-positive lymph nodes at (E) x100 and (F) x400 magnification. Positive staining of tumor cells was sporadically distributed. H\&E, hematoxylin and eosin; IHC, immunohistochemistry; CK, cytokeratin.

for the detection of human CK7 and CK8 as the next analytical step. From this analysis, human-derived epithelial tumor cells were successfully identified. Analysis of human CK8 expression revealed the presence of nesting-like positive cells (Fig. 4C and D). In addition, analysis of human CK7, which is used to characterize epithelial tumors (19), revealed the presence of sporadic strongly positive cells (Fig. 4E and F), suggesting that these lymph nodes contained metastatic ovarian tumor cells.

\section{Discussion}

The aim of the present study was to develop a fluorescent label for the analysis of lymphatic metastasis in vivo using fluorescently-labeled ovarian cancer cells. Imaging results of the cells in vitro revealed the presence of mCherry fluorescence. In addition, metastatic retroperitoneal lymph nodes were successfully identified in vivo by co-localization with lymph nodes labeled by NIR nanoparticles.

Of the metastatic lymph nodes, para-aortic and pelvic lymph nodes are the most common in patients with ovarian cancer. Furthermore, lymphogenous metastasis is associated with disease progression and recurrence $(7,20)$. Cytoreductive surgery is the standard approach for the management of ovarian cancer, and systematic lymphadenectomy is frequently conducted despite the associated complications and complexity of the surgical procedures $(8,9)$. However, isolation of a specific single node as the sentinel node is difficult prior to or during surgery (21).

It may be possible to apply positron emission tomography (PET)/computed tomography as a pre-operative imaging step in order to address this clinical dilemma. In ovarian cancer, few studies have examined the accuracy of these imaging 
modalities for the detection of metastasis (22). Owing to the spatial resolution of current PET scanners (4-6 mm) (23), lymph nodes $<5 \mathrm{~mm}$ in diameter may not be identified in early-stage ovarian cancer (22). In recurrent cases, a size threshold of $0.5 \mathrm{~cm}$ and $41 \%$ detection sensitivity of retroperitoneal lymph node metastases have been reported $(24,25)$. As a result, the detection of metastatic lymph nodes $<5 \mathrm{~mm}$ in diameter is impracticable with PET; thus, several imaging agents have been developed for the visualization of early or recurrent ovarian tumors.

Folate receptor (FR) $\alpha$ and human epidermal growth factor receptor (HER)-2 are widely used as targeting agents. $\mathrm{FR} \alpha$-targeted fluorescent agents have been applied experimentally in intra-operative imaging to facilitate staging and accurate radical cytoreductive surgery (26). This method has demonstrated promising results in histopathologically confirmed tumor deposits measuring $<1 \mathrm{~mm}$ for up to $8 \mathrm{~h}$ following injection. However, this study only addressed the peritoneal tumor deposits and did not report the capacity for detection of lymphatic systems (26). HER-2-targeted nanoparticles and low-molecular-weight proteins have also been employed as imaging agents in tumor-bearing mice in optical or PET imaging (27-29). Early-stage tumors with diameters of 1-2 mm were detectable in orthotopic ovarian tumor xenografts and small metastatic lesions $(\sim 1 \mathrm{~mm})$ in the peritoneal cavity and in lung metastasis (27). However, this detection was limited to HER-2-overexpressing SKOV-3 tumors, and lymphatic involvement has not been observed or investigated.

Application of NIR fluorescence imaging, which utilizes contrast agents with fluorescent characteristics in the NIR spectrum (700-900 nm), has emerged to allow researchers to overcome problems with anatomical guidance and tumor identification, particularly for minimally invasive surgeries. Identification of the vasculature, biliary tract in cholecystectomy, adrenal glands in partial adrenalectomy and nerves in thymectomy, as well as real-time visualization of ureters and tissue perfusion assessment in colorectal anastomosis, have been facilitated by navigation with NIR fluorescence imaging (30). For tumor delineation, NIR fluorescence agents targeting FR $\alpha$ and $\alpha v \beta 3$ integrin have been reported in ovarian cancer (31). Targeted $\alpha v \beta 3$ integrin imaging of intraperitoneal tumor xenografts has been demonstrated to have $100 \%$ sensitivity, $88 \%$ specificity and a mean target to background ratio of 2.2 (31). However, detection of lymphatic metastasis using this method has not been reported. NIR fluorescence agents have also been studied in sentinel lymph node mapping; The Food and Drug Administration-approved indocyanine green (ICG; $800 \mathrm{~nm}$ ) is the most common agent used in this context, and targeted contrast agents are not required when ICG is used (30). The feasibility and accuracy of sentinel lymph node mapping using ICG has been demonstrated in melanoma, colorectal cancer, breast cancer, non-small cell lung cancer, esophageal cancer, and gastric cancer (32-37). Furthermore, NIR fluorescence-based sentinel lymph node mapping has been studied in vulvar cancer, cervical cancer, and endometrial cancer (38-40); but few studies concerning sentinel lymph node mapping in ovarian cancer have been reported. Therefore, based on the above-mentioned studies, there is a need for less invasive and highly sensitive detection of lymphatic metastasis in ovarian cancer.

In the present study, nanoparticles were used for highly efficient lymph node labeling. Major lymph nodes, including the mandibular lymph nodes, superficial parotid lymph nodes, subiliac lymph nodes, and retroperitoneal medial iliac lymph nodes were identified $24 \mathrm{~h}$ following injection (excitation/emission: 465/780 nm). Identification of the lymph nodes was matched with the anatomy of murine lymph nodes (41). In addition, metastatic tumor cells were labeled with mCherry fluorescent protein; a general-purpose red fluorescent protein with superior photostability (42). As mCherry has a separate excitation/emission peak of 587/610 nm, it was possible to co-localize the metastatic lymph nodes where the two signals overlapped. Instead of using a targeting receptor, for example FR $\alpha$, HER-2 or $\alpha v \beta 3$-integrin, the metastatic tumors were successfully located based on the spatial overlapping of tumor signals and lymph node signals, allowing tumor delineation regardless of the expression of target receptors. Furthermore, with the identification of spatial overlapping signals, it is also possible to apply this method to track the metastatic tumors in the lymphatic system in time-course studies, which provide additional insights into the mechanisms of lymphatic metastasis. While routine $\mathrm{H} \& \mathrm{E}$ staining of these imaging-positive lymph nodes yielded histopathologically negative results, IHC analysis successfully identified human-derived epithelial tumor cells, demonstrating the high sensitivity of the imaging model of the present study for metastatic ovarian cancer lymph nodes. Thus, this method may contribute to the identification of metastatic lymph nodes when positive signals are presented and a more proactive pathological analysis is required. With further investigation, this model may be used for intra-operative imaging due to its potential for the sensitive identification of lymphatic metastasis, and it may also facilitate sentinel lymph node sampling in further studies. Furthermore, simple nanoparticles were successfully delivered to the metastatic lymph nodes, providing the opportunity for packaging of chemotherapy agents and delivery of these agents to metastatic lymphatic lesions.

The limitations of the present study include the potentially shallow tissue penetration depth of the imaging modality and the interference of autofluorescence, which is frequently observed in fluorescent signal analyses. Because of the interference of autofluorescence, all of the images were collected subsequent to the removal of the gastrointestinal and reproductive system tissues, and with black sheets covering the surrounding tissues.

In the present study, metastatic retroperitoneal lymph nodes were identified by co-localization of lymph nodes and tumor cells with NIR fluorescence nanoparticles in a xenograft mouse model. The results suggested that this method may yield important insights, and that it may be possible to apply these data to intra-operative identification of lymphatic metastasis, analysis of the mechanisms of lymphatic metastasis and evaluation of lymphatic drug delivery.

\section{Acknowledgements}

The present study was supported by The National Natural Science Foundation of China (grant no. 81472423); The 
Shanghai Natural Science Foundation of China (grant no. 13ZR1404300); The Experimental Animal Special Fund of Science and Technology Commission of Shanghai (grant no. 13140901500); Chinese Natural Science Foundation project (grant nos. 81301261 and 21374059); Key Project for Major Diseases of Health System of Shanghai (grant no. 2013ZYJB0201) and National Key Research and Development Program (grant nos. 2016YFC1303100, 2016YFC1303101, 2016YFC1303102 and 2016YFC1303103).

\section{References}

1. Siegel RL, Miller KD and Jemal A: Cancer statistics, 2015. CA Cancer J Clin 65: 5-29, 2015.

2. Lengyel E: Ovarian cancer development and metastasis. Am J Pathol 177: 1053-1064, 2010.

3. Tan DS, Agarwal R and Kaye SB: Mechanisms of transcoelomic metastasis in ovarian cancer. Lancet Oncol 7: 925-934, 2006

4. Gadducci A, Cosio S, Zola P, Sostegni B, Ferrero AM, Teti G, Cristofani R and Sartori E: The clinical outcome of epithelial ovarian cancer patients with apparently isolated lymph node recurrence: A multicenter retrospective Italian study. Gynecol Oncol 116: 358-363, 2010.

5. Mutch DG and Prat J: 2014 FIGO staging for ovarian, fallopian tube and peritoneal cancer. Gynecol Oncol 133: 401-404, 2014.

6. Kleppe M, Wang T, Van Gorp T, Slangen BF, Kruse AJ and Kruitwagen RF: Lymph node metastasis in stages I and II ovarian cancer: A review. Gynecol Oncol 123: 610-614, 2011.

7. Pereira A, Magrina JF, Rey V, Cortes M and Magtibay PM: Pelvic and aortic lymph node metastasis in epithelial ovarian cancer. Gynecol Oncol 105: 604-608, 2007.

8. Camara O and Sehouli J: Controversies in the management of ovarian cancer-pros and cons for lymph node dissection in ovarian cancer. Anticancer Res 29: 2837-2843, 2009.

9. Trimbos JB: Lymphadenectomy in ovarian cancer: Standard of care or unnecessary risk. Curr Opin Oncol 23: 507-511, 2011.

10. di Re F, Baiocchi G, Fontanelli R, Grosso G, Cobellis L, Raspagliesi F and di Re E: Systematic pelvic and paraaortic lymphadenectomy during cytoreductive surgery in advanced ovarian cancer: Potential benefit on survival. Gynecol Oncol 62: 360-365, 1996

11. Scarabelli C, Gallo A, Zarrelli A, Visentin C and Campagnutta E: Systematic pelvic and para-aortic lymphadenectomy during cytoreductive surgery in advanced ovarian cancer: Potential benefit on survival. Gynecol Oncol 56: 328-337, 1995.

12. Di Re F and Baiocchi G: Value of lymph node assessment in ovarian cancer: Status of the art at the end of the second millennium. Int J Gynecol Cancer 10: 435-442, 2000.

13. Shaw TJ, Senterman MK, Dawson K, Crane CA and Vanderhyden BC: Characterization of intraperitoneal, orthotopic and metastatic xenograft models of human ovarian cancer. Mol Ther 10: 1032-1042, 2004.

14. Ricci F, Broggini M and Damia G: Revisiting ovarian cancer preclinical models: Implications for a better management of the disease. Cancer Treat Rev 39: 561-568, 2013.

15. Lengyel E, Burdette JE, Kenny HA, Matei D, Pilrose J, Haluska P, Nephew KP, Hales DB and Stack MS: Epithelial ovarian cancer experimental models. Oncogene 33: 3619-3633, 2014.

16. Servais EL, Colovos C, Bograd AJ, White J, Sadelain M and Adusumilli PS: Animal models and molecular imaging tools to investigate lymph node metastases. J Mol Med (Berl) 89: 753-769, 2011

17. Kang Y, Pu T, Cai Q, Hong S, Zhang M, Li G, Zhu Z and Xu C: Identification of lymphatic metastasis associated genes in a metastatic ovarian cancer cell line. Mol Med Rep 12: 2741-2748, 2015.

18. Xiong L, Shuhendler AJ and Rao J: Self-luminescing BRET-FRET near-infrared dots for in vivo lymph-node mapping and tumour imaging. Nat Commun 3: 1193, 2012.

19. Painter JT, Clayton NP and Herbert RA: Useful immunohistochemical markers of tumor differentiation. Toxicol Pathol 38: 131-141, 2010.

20. Pereira A, Pérez-Medina T, Magrina JF, Magtibay PM, Rodríguez-Tapia A, de León J, Peregrin I and Ortiz-Quintana L: Correlation between the extent of intraperitoneal disease and nodal metastasis in node-positive ovarian cancer patients. Eur J Surg Oncol 40: 917-924, 2014.
21. Classe JM, Cerato E, Boursier C, Dauplat J, Pomel C, Villet R, Cuisenier J, Lorimier G, Rodier JF, Mathevet P, et al: Retroperitoneal lymphadenectomy and survival of patients treated for an advanced ovarian cancer: The CARACO trial. J Gynecol Obstet Biol Reprod (Paris) 40: 201-204, 2011 (In French).

22. Signorelli M, Guerra L, Pirovano C, Crivellaro C, Fruscio R, Buda A, Cuzzucrea M, Elisei F, Ceppi L and Messa C: Detection of nodal metastases by 18F-FDG PET/CT in apparent early stage ovarian cancer: A prospective study. Gynecol Oncol 131: 395-399, 2013.

23. Kitajima K, Murakami K, Yamasaki E, Kaji Y, Fukasawa I, Inaba $\mathrm{N}$ and Sugimura K: Diagnostic accuracy of integrated FDG-PET/contrast-enhanced CT in staging ovarian cancer: Comparison with enhanced CT. Eur J Nucl Med Mol Imaging 35: 1912-1920, 2008

24. Bristow RE, Giuntoli RL II, Pannu HK, Schulick RD, Fishman EK and Wahl RL: Combined PET/CT for detecting recurrent ovarian cancer limited to retroperitoneal lymph nodes. Gynecol Oncol 99: 294-300, 2005.

25. Pannu HK, Cohade C, Bristow RE, Fishman EK and Wahl RL: PET-CT detection of abdominal recurrence of ovarian cancer: Radiologic-surgical correlation. Abdom Imaging 29: 398-403, 2004.

26. van Dam GM, Themelis G, Crane LM, Harlaar NJ, Pleijhuis RG, Kelder W, Sarantopoulos A, de Jong JS, Arts HJ, van der Zee AG, et al: Intraoperative tumor-specific fluorescence imaging in ovarian cancer by folate receptor-alpha targeting: First in-human results. Nat Med 17: 1315-1319, 2011.

27. Satpathy M, Wang L, Zielinski R, Qian W, Lipowska M, Capala J, Lee GY, Xu H, Wang YA, Mao H and Yang L: Active targeting using HER-2-affibody-conjugated nanoparticles enabled sensitive and specific imaging of orthotopic HER-2 positive ovarian tumors. Small 10: 544-555, 2014.

28. Ren G, Webster JM, Liu Z, Zhang R, Miao Z, Liu H, Gambhir SS, Syud FA and Cheng Z: In vivo targeting of HER2-positive tumor using 2-helix affibody molecules. Amino Acids 43: 405-13, 2012.

29. Miao Z, Ren G, Jiang L, Liu H, Webster JM, Zhang R, Namavari M, Gambhir SS, Syud F and Cheng Z: A novel 18F-labeled two-helix scaffold protein for PET imaging of HER2-positive tumor. Eur J Nucl Med Mol Imaging 38: 1977-1984, 2011.

30. Schols RM, Connell NJ and Stassen LP: Near-infrared fluorescence imaging for real-time intraoperative anatomical guidance in minimally invasive surgery: A systematic review of the literature. World J Surg 39: 1069-1079, 2015.

31. Harlaar NJ, Kelder W, Sarantopoulos A, Bart J, Themelis G, van Dam GM and Ntziachristos V: Real-time near infrared fluorescence (NIRF) intra-operative imaging in ovarian cancer using an $\alpha(\mathrm{v}) \beta(3-)$ integrin targeted agent. Gynecol Oncol 128: 590-595, 2013.

32. van der Vorst JR, Schaafsma BE, Verbeek FP, Swijnenburg RJ, Hutteman M, Liefers GJ, van de Velde CJ, Frangioni JV and Vahrmeijer AL: Dose optimization for near-infrared fluorescence sentinel lymph node mapping in patients with melanoma. Br J Dermatol 168: 93-98, 2013.

33. Cahill RA, Anderson M, Wang LM, Lindsey I, Cunningham C and Mortensen NJ: Near-infrared (NIR) laparoscopy for intraoperative lymphatic road-mapping and sentinel node identification during definitive surgical resection of early-stage colorectal neoplasia. Surg Endosc 26: 197-204, 2012.

34. Troyan SL, Kianzad V, Gibbs-Strauss SL, Gioux S, Matsui A, Oketokoun R, Ngo L, Khamene A, Azar F and Frangioni JV: The FLARE intraoperative near-infrared fluorescence imaging system: A first-in-human clinical trial in breast cancer sentinel lymph node mapping. Ann Surg Oncol 16: 2943-2952, 2009.

35. Yamashita S, Tokuishi K, Miyawaki M, Anami K, Moroga T, Takeno S, Chujo M, Yamamoto S and Kawahara K: Sentinel node navigation surgery by thoracoscopic fluorescence imaging system and molecular examination in non-small cell lung cancer. Ann Surg Oncol 19: 728-733, 2012.

36. Kubota K, Yoshida M, Kuroda J, Okada A, Ohta K and Kitajima M: Application of the hypereye medical system for esophageal cancer surgery: A preliminary report. Surg Today 43: 215-220, 2013.

37. Tajima Y, Murakami M, Yamazaki K, Masuda Y, Kato M, Sato A, Goto S, Otsuka K, Kato T and Kusano M: Sentinel node mapping guided by indocyanine green fluorescence imaging during laparoscopic surgery in gastric cancer. Ann Surg Oncol 17: 1787-1793, 2010. 
38. Hutteman M, van der Vorst JR, Gaarenstroom KN, Peters AA, Mieog JS, Schaafsma BE, Löwik CW, Frangioni JV, van de Velde CJ and Vahrmeijer AL: Optimization of near-infrared fluorescent sentinel lymph node mapping for vulvar cancer. Am J Obstet Gynecol 206: 89. e1-e5, 2012.

39. van der Vorst JR, Hutteman M, Gaarenstroom KN, Peters AA, Mieog JS, Schaafsma BE, Kuppen PJ, Frangioni JV, van de Velde CJ and Vahrmeijer AL: Optimization of near-infrared fluorescent sentinel lymph node mapping in cervical cancer patients. Int J Gynecol Cancer 21: 1472-1478, 2011.
40. Holloway RW, Bravo RA, Rakowski JA, James JA, Jeppson CN Ingersoll SB and Ahmad S: Detection of sentinel lymph nodes in patients with endometrial cancer undergoing robotic-assisted staging: A comparison of colorimetric and fluorescence imaging. Gynecol Oncol 126: 25-29, 2012.

41. Van den Broeck W, Derore A and Simoens P: Anatomy and nomenclature of murine lymph nodes: Descriptive study and nomenclatory standardization in BALB/cAnNCrl mice. J Immunol Methods 312: 12-19, 2006.

42. Shaner NC, Steinbach PA and Tsien RY: A guide to choosing fluorescent proteins. Nat Methods 2: 905-909, 2005. 